\title{
Tranexamic acid has no influence on cardioprotection induced by ischaemic preconditioning or remote ischaemic preconditioning
}

\author{
${ }^{1}$ F. Behmenburg, ${ }^{1}$ M. Dorsch, ${ }^{1}$ P. van Caster, ${ }^{1}$ S. Eiling, ${ }^{2}$ M.W. Hollmann, ${ }^{1}$ R. Huhn \\ 1Department of Anaesthesiology, University Hospital Duesseldorf, Germany \\ ${ }^{2}$ Department of Anesthesiology, Laboratory of Experimental Intensive Care and Anesthesiology (L.E.I.C.A.), Academic Medical Center \\ (AMC), University of Amsterdam, The Netherlands
}

\section{Background and Goal of Study}

Ischaemic Preconditioning (IPC) and Remote Ischaemic Preconditioning (RIPC) reduce ischaemia/reperfusion injury in experimental animal studies [1]. In contrast, clinical data are contradictive. This might be caused by interactions of drugs used in daily clinical routine. The antifibrinolytic Aprotinin, a precursor of the currently used tranexamic acid (TXA), was shown to abolish cardioprotection by preconditioning [2]. The aim of this study was to investigate, whether TXA has an impact on cardioprotection induced by IPC and/or RIPC.

\section{Material and Methods}

Animals were treated in compliance with institutional and national guidelines. Anaesthetized Wistar rats were thoracotomized and, after pericardiotomy, a snare occluder was passed around a major left coronary artery. After surgical preparation, animals recovered for 20 minutes before starting the preconditioning protocol. Subsequently, rats were exposed to 25 minutes regional myocardial ischemia followed by 120 minutes reperfusion. At the end of reperfusion, hearts were excised for infarct size measurement by TTC staining.

Animals were randomized into six groups. Control animals (Con, $n=10)$ were not further treated. IPC was induced by 3 cycles of 5 minutes of ischaemia and reperfusion (IPC, $n=8$ ); RIPC was induced by 4 cycles of 5 minutes of ischaemia and reperfusion of the hind legs (RIPC, $n=9$ ). In additional groups tranexamic acid (bolus $10 \mathrm{mg} / \mathrm{kg} \mathrm{BW}$, continuously $2 \mathrm{mg} / \mathrm{kg}$ BW) was administered i.v. with (TXA+IPC, TXA+RIPC; each group $n=8$ ) and without preconditioning (TXA, $n=8)$.

Statistical analysis: One-way ANOVA with Tukey post hoc test. Data are expressed as mean \pm SD.

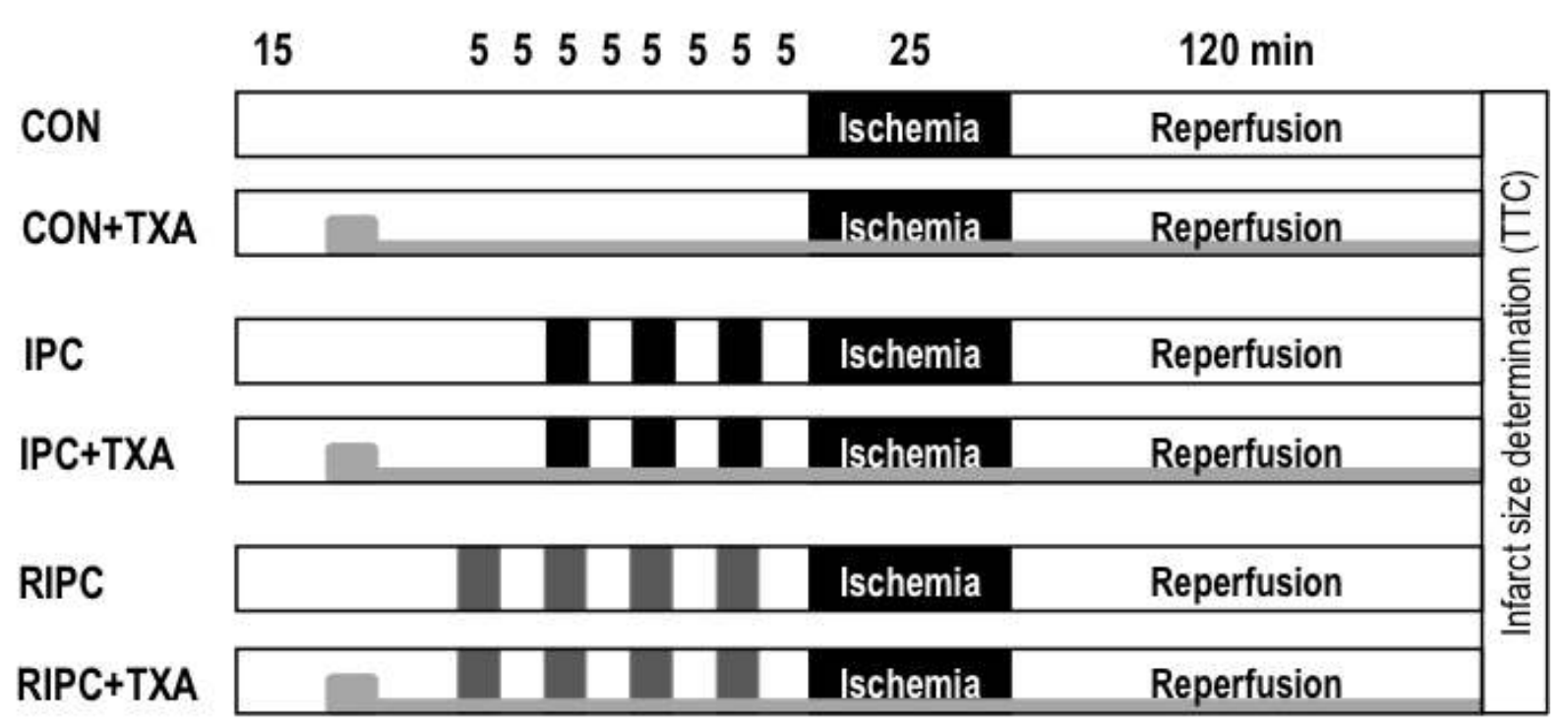

\section{Results}

In the control group infarct size was $56 \pm 11 \%$ of area at risk. IPC and RIPC reduced infarct size to $30 \pm 6 \%$ and $40 \pm 8 \%$, respectively (each $\mathrm{P}<0.05$ vs. Con). Tranexamic acid alone had no influence on infarct size (TXA: $54 \pm 15 \%$; ns vs. Con). Furthermore, tranexamic acid did not affect cardioprotection by IPC or RIPC (TXA+IPC: $25 \pm 8 \%$, TXA+RIPC: $41 \pm 10 \%$; each $P<0.05$ vs. Con).

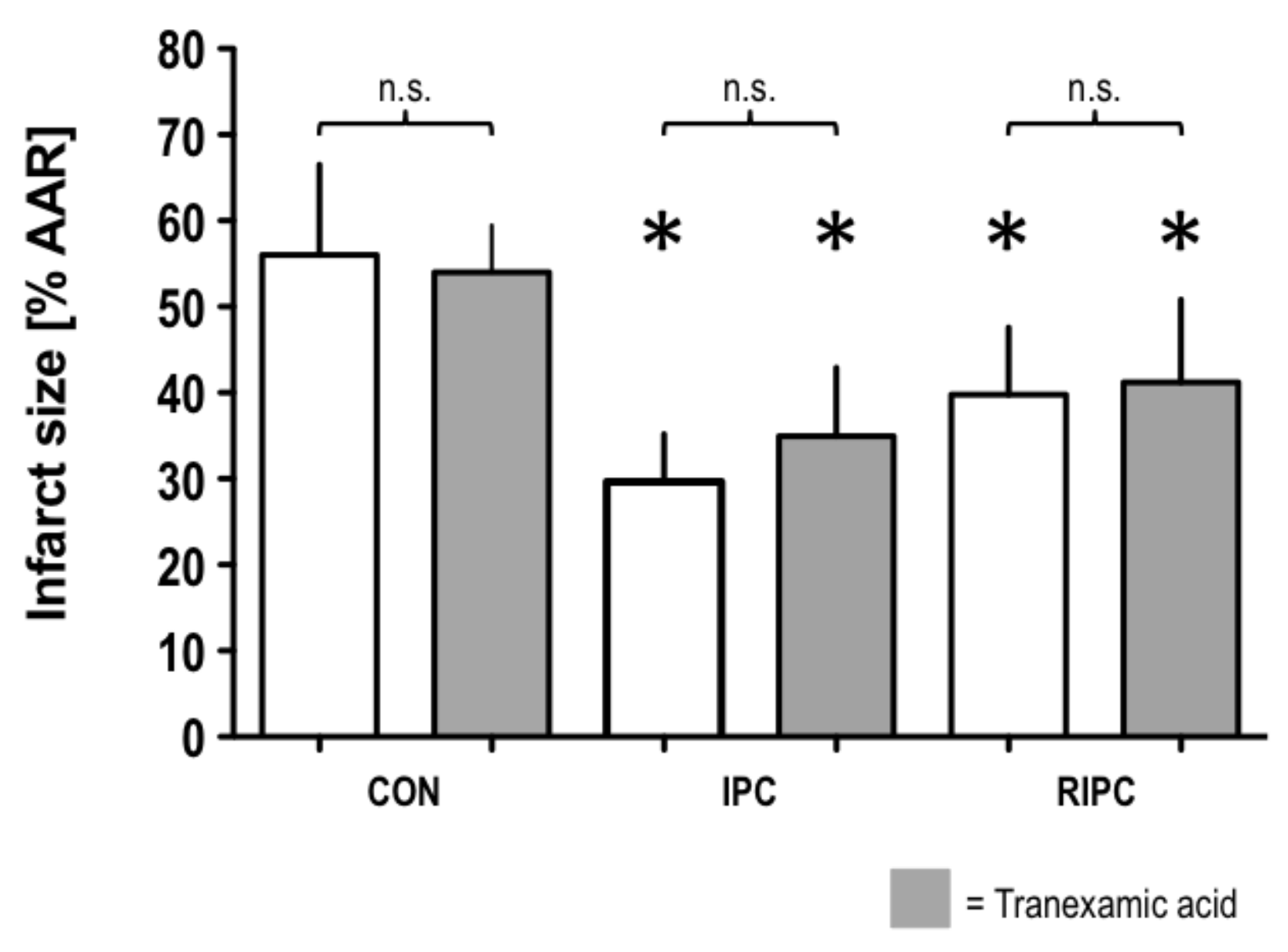

Fig.2 Infarct sizes (percent of area at risk, AAR) of controls (CON), ischemic preconditioning (IPC), remote ischemic preconditioning (RIPC), without or with continuous application of tranexamic acid.

\section{Conclusion}

Administration of tranexamic acid had no effect on myocardial infarction or the cardioprotective effects of ischaemic preconditioning or remote ischaemic preconditioning.

\section{Literature}

[1] Pharmacol Rev. 2014 Oct;66(4):1142-74,

[2] Anesthesiology $2010 \mathrm{Dec} ; 113(6): 1289-98$. 